\title{
AVALIAÇÃO COMPARATIVA DO DESEMPENHO DE ALUNOS DO ENSINO BÁSICO ACERCA DAS HABILIDADES DO PENSAMENTO COMPUTACIONAL
}

\author{
Ravenna Lins Rodrigues (UNIVERSIDADE FEDERAL DE CAMPINA GRANDE) \\ ravennalinsrodrigues@gmail.com \\ Cecir Barbosa de Almeida Farias (UNIVERSIDADE FEDERAL DE CAMPINA GRANDE) \\ cecir.almeida@gmail.com
}

\begin{abstract}
Resumo
O pensamento computacional tem sido considerado uma das principais competências para prosperar em um mundo tecnológico, e diversas iniciativas têm surgido visando o seu desenvolvimento desde a educação básica. Sua inserção nas escolas possibilita a aquisição de habilidades necessárias à resolução de problemas, podendo apoiar e relacionar-se com outras ciências. Nesse contexto o presente trabalho objetivou quantificar o desempenho prévio e posterior ao curso "ProgramAção" de alunos pertencentes ao ensino médio, mediante a aplicação de teste. O referido teste, foi desenvolvido por Román-González (2015), este é composto por 28 questões, as quais tenta identificar a habilidade de formação e solução de problemas, baseando-se nos conceitos fundamentais da Computação. Apesar do tamanho da amostra não ser estatisticamente significativo, os resultados apontam a eficácia da oficina em relação à aprendizagem de conteúdos de computação o que pode ser usado de forma a melhorar o ensino da computação na Educação Básica.
\end{abstract}

Palavras-Chaves: Ensino, Metodologia ativa, Programação em blocos, Pensamento computacional, App Inventor.

\section{Introdução}

As incessantes mudanças na sociedade e a entrada de sistemas computacionais cada vez mais complexos têm trazido consigo desafios que demandam novas abordagens para serem solucionados.

Os indivíduos em sua interação social cotidiana são diariamente desafiados a resolver problemas complexos, exigindo habilidade de correlacionar conceitos e categorias de diferentes naturezas e disciplinas. Dessa forma, a escola enquanto espaço de produção e disseminação de conhecimento tem procurado se posicionar perante essa realidade e prover ações educacionais mais pertinentes às exigências sociais da contemporaneidade.

Países como Israel (Gal-ezer, 1999), Canadá (MEO, 2008), Estados Unidos (CSTA, 2005) e Inglaterra (Csizmadia et al. 2015) têm defendido o ensino dos fundamentos da computação na educação básica com o intuito de desenvolver nos estudantes uma maneira computacional de pensar, modelar e resolver problemas de diversas áreas. 
No Brasil diversas iniciativas vêm sendo realizadas em diferentes regiões do país e publicadas em anais de eventos do Congresso Brasileiro de Informática na Educação (CBIE), como o Workshop de Ensino em Pensamento Computacional, Algoritmos e Programação (WAlgProg). Há ainda aquelas publicadas nos anais do Workshop sobre Educação em Computação (WEI) e em periódicos como a Revista Brasileira de Informática na Educação (RBIE) e a Revista Novas Tecnologias na Educação (RENOTE).

É possível observar que inúmeras ações de incluir conceitos fundamentais de computação - conhecimentos de lógica de programação, estruturação de algoritmos e a abstração necessária à resolução de problemas computacionais - vêm sendo propostas para os currículos do ensino fundamental e médio (Fernandes e Menezes 2011; Marques et al. 2011; Rapkiewicz et al. 2006).

Atualmente, pesquisas em Pensamento computacional, apresentam propostas curriculares e cursos de curta duração para o desenvolvimento ou inserção do PC no nível básico de ensino e acredita-se que essas habilidades podem contribuir para o desenvolvimento cognitivo de estudantes estimulando capacidades como raciocínio lógico, abstração e resolução de problemas, consideradas hoje como fundamentais para a formação básica.

Neste contexto, o pensamento computacional desponta como uma forma de resolver problemas, conceber sistemas e compreender o comportamento humano que se baseia em conceitos fundamentais da Ciência da Computação. Sua aplicabilidade para resolução de problemas nos mais diversos campos do conhecimento o torna uma habilidade fundamental para todas as pessoas, não apenas para cientistas da computação, despontando como um requisito elementar para a formação básica dos profissionais de todas as áreas nos próximos anos (Wing, 2006)

Existem diversas maneiras de desenvolver o pensamento computacional, uma delas é por meio de atividades práticas de programação, como as atividades da Hour of Code (code.org) ou por meio do desenvolvimento de jogos, animações e aplicativos pelos alunos (Grover e Pea 2013; Lye e Koh 2014; Ortiz e Pereira 2018; Santos et al. 2018). Para esse fim, tipicamente se adotam linguagens de programação visual (Santos et al. 2018; Dagostini et al. 2018), como, por exemplo, App Inventor para desenvolver aplicativos móveis (Daniel et al. 2017).

Diante desse cenário, buscou-se avaliar e quantificar o desempenho de alunos inscritos na oficina "ProgramAção", por intermédio de, pré teste o qual visou identificar os conhecimentos prévios dos alunos e pós teste onde este por sua vez buscou investigar se houve aumento no desempenho dos alunos. 


\section{Referencial Teórico}

O desenvolvimento das habilidades de resolução de problemas e de senso crítico é necessário frente às transformações sociais e a crescente competitividade no mundo atual. A inclusão do Pensamento Computacional no âmbito escolar contribui na vida social e escolar do estudante, pois, a partir do aprimoramento dessa habilidade, ele pode enfrentar seus desafios diários com maior facilidade (Valente, 2016).

O pensamento computacional (do inglês, computational thinking) engloba métodos para solução de problemas baseado nos fundamentos e técnicas da Ciência da Computação, e é visto como uma das formas de desenvolver o raciocínio lógico. Neste sentido, por meio do desenvolvimento de tais fundamentos, o aluno poderá desenvolver técnicas como abstração, organização e execução passo a passo para resolução de problemas, o que irá auxiliá-lo na elaboração do seu pensamento (KRAMER, 2007). Além disso, a partir de tais conceitos, o estudante deixará de compreender o computador como mero artefato tecnológico, mas como um potencializador de suas atividades.

A escola tem um papel significativo na formação dos indivíduos, na sua cultura, nas suas relações sociais e, decididamente, em seu processo de interpretação da informação. É uma instituição que reúne condições propícias para desempenhar esse papel. Na escola, os alunos podem ser apresentados a um conjunto organizado e planejado de temas e situações de aprendizagem que pode ser sistematizado gerando oportunidades para que sejam autores do próprio conhecimento.

Waiselfisz (2007) considera que é importante que o educando se desenvolva e se aproprie de propostas de autoria digital, com autonomia no pensar e no agir, sendo necessário ainda reconhecer o binômio ensino e aprendizagem como uma relação entre sujeitos, em que cada um, a seu modo e com determinado papel, está envolvido na construção de uma compreensão dos fenômenos naturais e suas transformações, na formação de novas atitudes e valores. Podemos, dessa forma, entender que o aluno se torna sujeito de sua aprendizagem quando é dele o movimento de dar novos significados ao mundo, o que pode ser entendido como construir explicações norteadas pelo pensamento computacional.

\section{Metodologia}

Desenvolveu-se um projeto pedagógico, no formato de curso introdutório de programação onde os procedimentos metodológicos utilizados foram: (a) Apresentação do 
projeto para os alunos dos ensino médio da Escola Estadual Professor José Gonçalves de Queiroz localizada no município de Sumé, região do cariri paraibano; (b) Inscrição dos alunos interessados em participar do curso; (c) Preparação do Laboratório de Informática da Universidade Federal de Campina Grande - Centro de Desenvolvimento Sustentável do Semiárido, para a execução das oficinas; (d) Aplicação de um questionário a fim de verificar o perfil dos participantes, o grau de conhecimento em computação e suas respectivas dificuldades; (e) Aplicação das aulas com a turma formada a partir dos alunos inscritos, pertencentes ao ensino médio; (f) Estímulo ao raciocínio lógico e criatividade por meio de atividades que visavam a criação de pequenos projetos de programação, partindo de trabalhos individuais e em equipes; (g) Aplicação de questionário ao término das oficinas, a fim de comparar se houve crescimento significativo no conhecimento sobre a computação.

\section{Fase Exploratória}

O curso denominado "ProgramAção", foi ministrado por bolsistas e extensionistas do Probex (O Programa de Bolsas de Extensão). A turma era composta por 22 alunos, . As aulas transcorreram a partir de encontros semanais, com duração de $2 \mathrm{~h} /$ aulas. $\mathrm{O}$ planejamento dessas aulas baseou-se nas seguintes etapas:

A $1^{\text {a }}$ etapa: concebeu a aplicação do questionário sobre pensamento computacional desenvolvido por Román González et al. (2015), o qual visou a identificação dos conhecimentos prévios dos alunos.

$2^{\text {a }}$ etapa: consistiu no ciclo mais duradouro de aulas, bem como o de maior relevância também, pois nele se conduziu o ensino de conceitos fundamentais de programação. Para cada conceito, foi apresentada a sua definição, exemplos de uso e, quando possível, a aplicação desses conceitos em outros contextos que não a Computação.

A $3^{\text {a }}$ etapa: consistiu no ensino do conceito de programação em blocos, abordando a sua lógica, seguida pela apresentação da plataforma App Inventor, que apresenta uma abordagem lúdica e de simples funcionamento.

$4^{\text {a }}$ etapa: consistiu na utilização da plataforma, de maneira prática, apresentando as ferramentas, recursos e componentes existentes. Nesse estágio os alunos puderam manipular menus, e telas, assimilando os elementos básicos presentes na programação. No decorrer deste processo, foi trabalhado atividades realizadas pelos alunos, na plataforma, com ajuda dos extensionista. 
A $5^{a}$ etapa: composta pelo desenvolvimento em grupo e individual de aplicações móveis, baseadas no conhecimento adquiridos na etapa anterior. Tais conhecimentos alicerçaram a produção em grupo de: Uma Calculadora de IMC e um Jogo de Ping Pong, ambos elaborados de forma independente entre os alunos. Esta metodologia baseada na criação de pequenos projetos desenvolvidos ao longo do curso, proporcionou uma maior interação com a linguagem de programação, bem como o melhor acompanhamento da aprendizagem. Em todas atividades propostas, trabalhou-se a aprendizagem colaborativa para a criação dos aplicativos propostos, facilitando a troca de conhecimentos através do trabalho em grupo. Entre conteúdos práticos e teóricos, estimulou-se a formação de alunos autodidatas, com o intuito de aprimorar a capacidade de raciocínio.

$6^{\text {a }}$ etapa: concebeu novamente a aplicação do questionário sobre pensamento computacional desenvolvido por Román González et al. (2015), este por sua vez buscou investigar se houve aumento no desempenho dos alunos, isto é, se os mesmos conseguiram assimilar os conteúdos abordados, e se de fato o uso da plataforma MIT App Inventor facilitou o aprendizado de lógica de programação.

Em harmonia com todas as etapas, fundamentou-se a análise de dados, a partir de uma abordagem quantitativa referente aos questionários iniciais e finais.

\section{Instrumento avaliativo}

O teste utilizado na pesquisa foi escolhido devido à sua abordagem quantitativa $\mathrm{e}$ aptitudinal e porque já sofreu um rigoroso processo de validação, que comprovou a validade do conteúdo (ROMÁN-GONZÁLEZ, 2015). Este teste tenta identificar a habilidade de formação e solução de problemas, baseando-se nos conceitos fundamentais da Computação, além de utilizar sintaxes lógicas usadas nas linguagens de programação. As questões que compõem o instrumento avaliativo incluem conceitos dos quatro pilares do Pensamento Computacional: abstração, decomposição, reconhecimento de padrões e algoritmos. $\mathrm{O}$ instrumento é composto por 28 questões de múltipla escolha, sendo que cada questão possui quatro alternativas de resposta e somente uma é válida.

\section{Resultados}

De forma geral o questionário inicial apresentou uma média de oito $(\sim 8,35 ; 36,98 \%)$ acertos, em relação ao questionário final que apresentou uma média de doze acertos e meio 
$(\sim 12,46 ; 56,65 \%)$ por questão referente a habilidade abstração do pensamento computacional. A Figura 1, mostra o desempenho médio dos alunos nas questão de abstração. $\mathrm{O}$ decréscimo das performances dos alunos deve-se a complexidade progressiva das questões.

Figura 1. Representação gráfica dos índice inicial e final de respostas corretas para cada questão referente a habilidade "abstração" do pensamento computacional

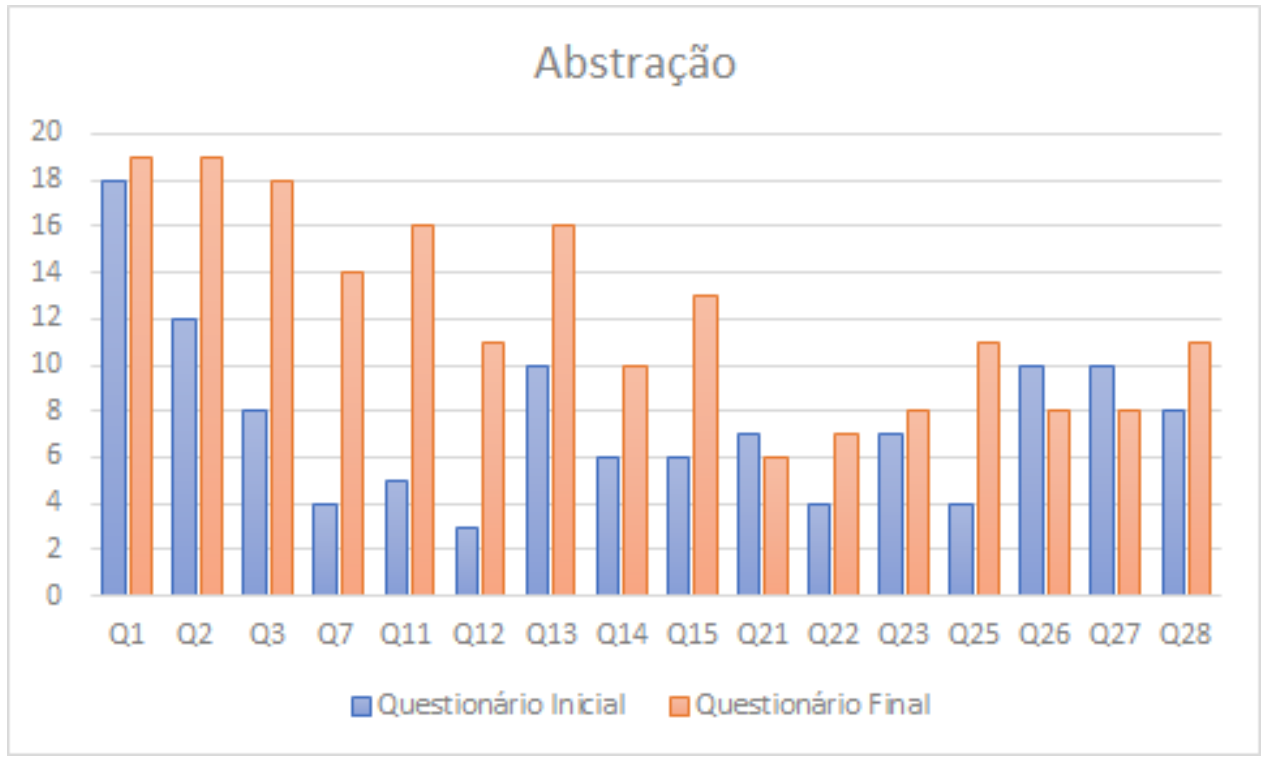

Fonte: Autor da Pesquisa

A avaliação da capacidade média de decomposição de problemas mostrou um avanço médio de 20,26 pontos percentuais dos alunos na capacidade de fragmentar grandes problemas em problemas menores. $\mathrm{O}$ incremento totalizou um percentual final de $58.86 \%$ como resultado médio de acertos desta categoria, como pode ser visto na Figura 2.

Figura 2. Representação gráfica dos índice inicial e final de respostas corretas para cada questão referente a habilidade "decomposição" do pensamento computacional 


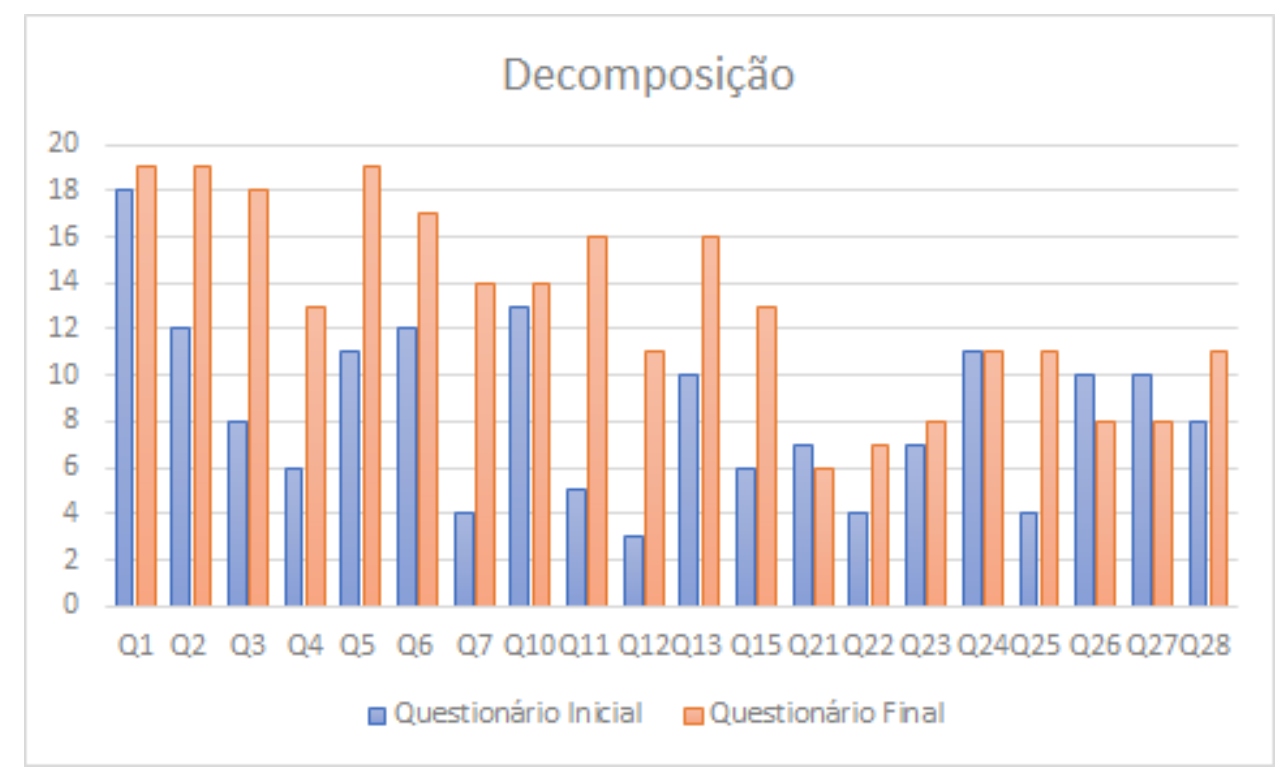

Fonte: Autor da Pesquisa

As questões referentes a reconhecimento de padrões apresentaram o menor percentual final médio de acertos pós o curso de programação em blocos. Entretanto, percebeu-se um incremento de 17,7 pontos percentuais à média inicialmente registrada nos alunos, que alcançaram a média de acertos global $>50 \%$ (53,8\%). A representação da relação entre os acertos médios inicial e final das questões de reconhecimento de padrões pode observada na Figura 3.

Figura 3. Representação gráfica dos índice inicial e final de respostas corretas para cada questão referente a habilidade "reconhecimento de padrões" do pensamento computacional

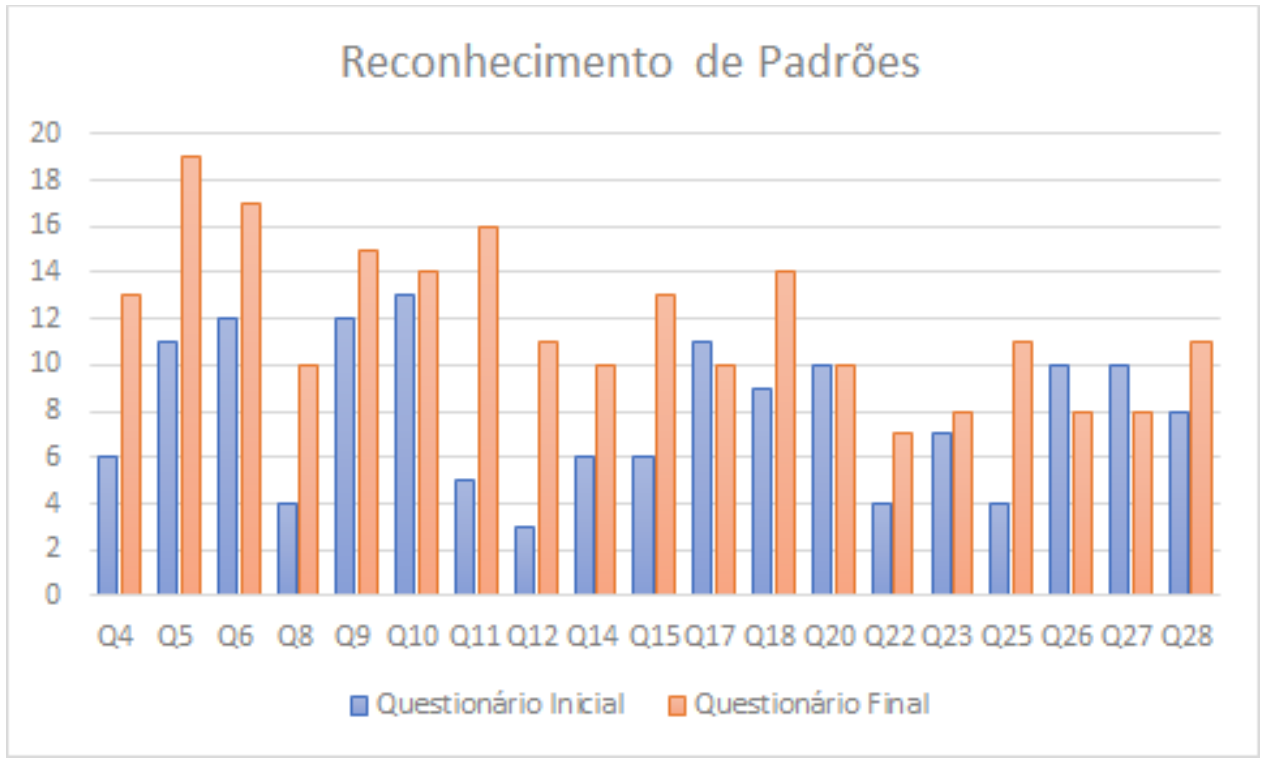


A capacidade de compreensão média dos alunos em relação à algoritmos foi representada pelas questões corretamente respondidas, e mediram um total de $37,98 \%$ de acertos nos gabaritos iniciais prévios ao curso. Esta média recebeu um incremento de 18,68 pontos percentuais, alcançando $56,65 \%$ de acertos.

Figura 4. Representação gráfica dos índice inicial e final de respostas corretas para cada questão referente a habilidade "algoritmo" do pensamento computacional

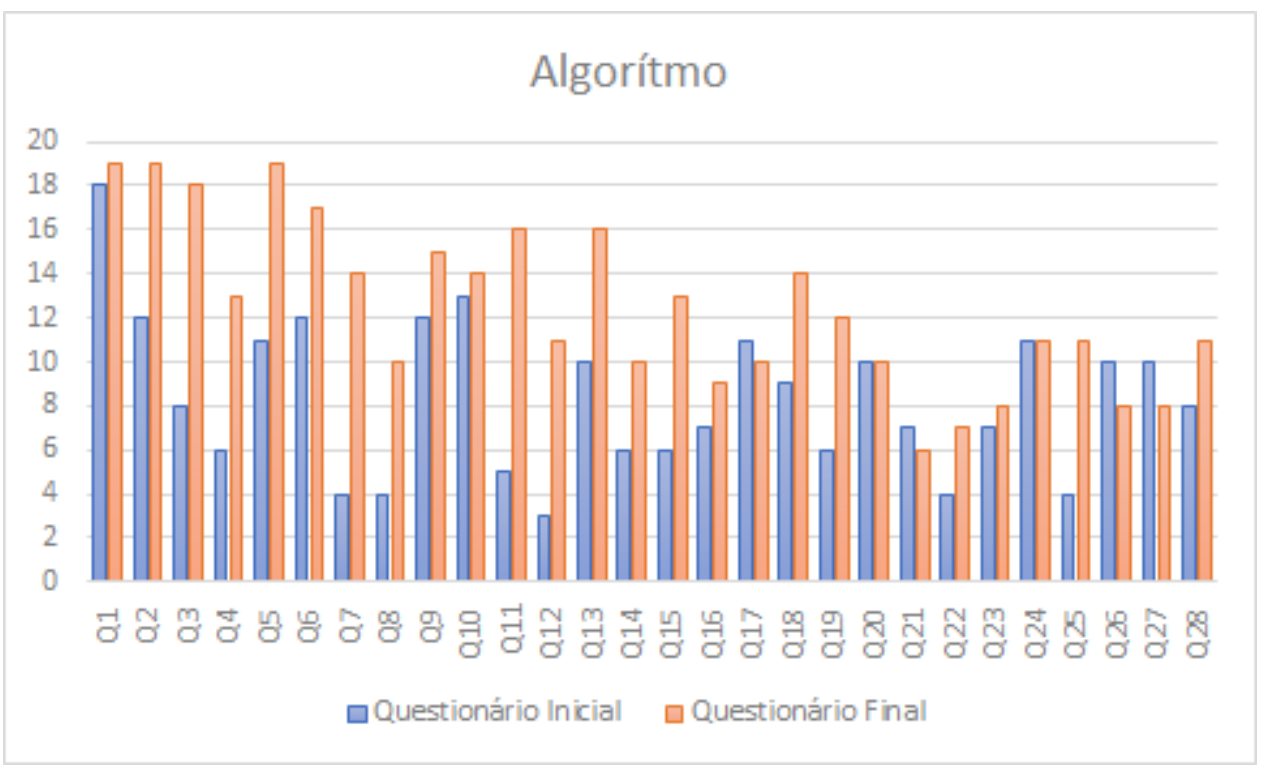

Fonte: Autor da Pesquisa

Foi constatado pelo teste de habilidades prévio ao curso de programação em blocos com o "AppInventor", apresentou, um percentual médio de acerto inferior a $40 \%$ por habilidade computacional. Tal que, é possível perceber (mediante a Figura 5), que houve um aumento de quatro acertos médios por questão de cada habilidade computacional, que totalizam um aumento aproximado médio de $20 \%$ de respostas corretas no resultado na avaliação final de cada habilidade.

Figura 5. Representação gráfica dos índice inicial e final de respostas corretas para cada habilidade do pensamento computacional 


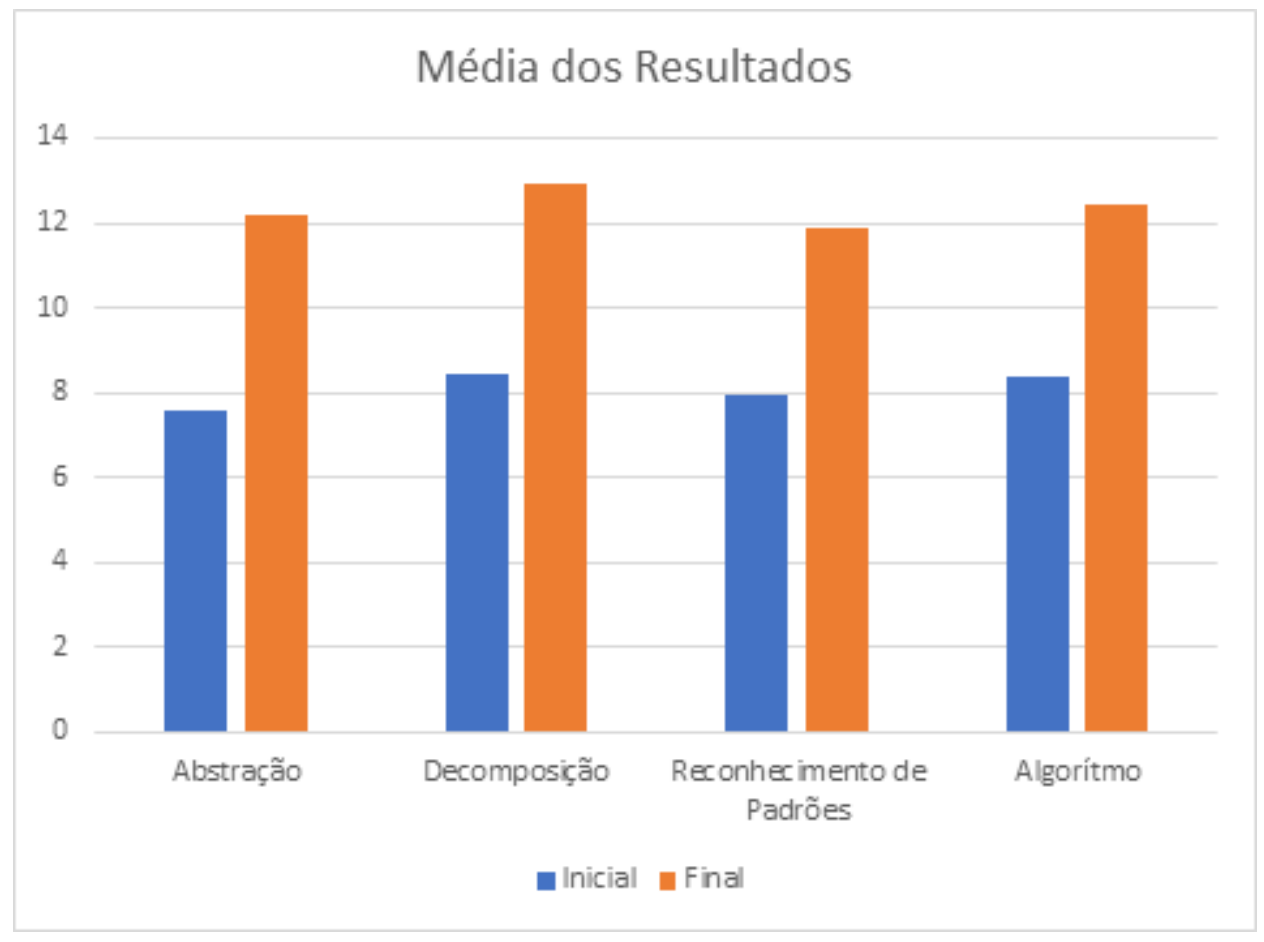

Fonte: Autor da Pesquisa

O número global médio de acertos por questão registrado no questionário inicial foi $\sim 8,085$, enquanto o registro final de acertos médios mostrou aproximadamente 12,36 acertos por questão. A capacidade média dos alunos de utilizar as habilidades "Abstração", "Decomposição", "Reconhecimento de Padrões" e "Algoritmos", para solucionar os problemas que lhes foram apresentados neste estudo foi notoriamente maior, pós estabelecidas as noções de programação em blocos proposta no curso. O incremento final médio de habilidade do pensamento computacional totalizou uma aproximação de 19,4\%, totalizando uma média global de aproximadamente 56,15/100.

\section{Conclusão}

A amostra de alunos selecionados para participar do curso de programação em blocos conseguiram ao decorrer do curso desenvolver as competências do pensamento computacional. Foi possível observar um aumento em cada uma das quatro habilidades do pensamento computacional. O questionário de Román-González (2015) se apresentou útil para a mensuração das competências do pensamento computacional. O AppInventor se apresentou 
uma boa ferramenta para o desenvolvimento de aulas práticas de programação em blocos. A programação em blocos se apresentou um recurso didático para o processo de ensino introdutório de programação.

\section{Referências}

Computer Science Teacher Association CSTA. (2005) The New Educational Imperative: Improving High School Computer Science Education. Final Report of the CSTA.Curriculum Improvement Task Force. ACM - Association for Computing Machinery

Csizmadia, A.; Curzon, P.; Dorling, M.; Humphreys, S.; Thomas Ng; Selby, C.; Woollard, J.. (2015). Computationalthinking: a guide for teachers. Computing At School. Gal-Ezer, J.; D. Harel.(1999) Curriculum for a high school computer science curriculum.Computer Science Education 9(2).

Dagostini et al. (2018) "URI Online Judge Blocks: Construindo Soluções em uma Plataforma Online de Programação”, In: Anais do Simpósio Brasileiro de Informática na Educação, Fortaleza, Brasil.

Daniel, G. T. et al. (2017) “Ensinando a Computação por meio de Programação com App Inventor”, In: Anais do Computer on the Beach, Florianópolis, p. 357-365.

Encyclopedia of Curriculum Studies, SAGE Publications. Lye, S. Y., Koh, J. H. L. (2014) "Review on teaching and learning of computational thinking through programming: What is next for K-12?" Computers in Human Behavior, v. 41(C), p. 51-61.

Fernandes, C. S.; Menezes, P. B. (2011) "Metodologia do Ensino de Ciência da Computação: Uma Proposta Para Criança”. In: Anais do Workshop de Informática na Escola. Fortaleza: CE.

Grover, S., Pea, R. (2013) "Computational Thinking in K-12 A review of the state of the field", Educational Researcher, v. 42, n. 1, p. 38-43.

Marques, D. L., Costa, L. F. S., Silva, M. A. A., Rebouças, A. D. D. S. (2011) "Atraindo Alunos do Ensino Médio para a Computação: Uma Experiência Prática de Introdução à Programação utilizando Jogos e Python”. In: Anais do XVII Workshop sobre Educação Informática. Aracaju: SE

KRAMER, Jeff. Is abstraction the key to computing? Communications of the ACM, v. 50, n. 4, p. 36-42, 2007.

MEC (2018) “Base Nacional Comum Curricular”, Brasil.

Ministério da Educação de Ontário - MEO. (2008). "Currículo para o ensino de Ciência da Computação nas escolas". Retirado de www.edu.gov.on.ca

Ortiz J. S. e Pereira R. (2018) "Um Mapeamento Sistemático Sobre as Iniciativas para Promover o Pensamento Computacional", In: Simpósio Brasileiro de Informática na Educação, Fortaleza, Brasil. 
Rapkiewicz, C. E., Falkembach, G., Seixas, L., Rosa, N. S., Cunha, V. V. Klemann, M. (2006) "Estratégias Pedagógicas no Ensino de Algoritmos e Programação Associadas ao Uso de Jogos Educacionais". In: CINTED-UFRGS, V. 4 N² 2.

Román-González, M. (2015). Computational thinking test: Design guidelines and content validation. EDULEARN15, pages 2436-2444.

Santos, P. S. C., Araujo, L. G. J., Bittencourt, R. A. (2018) “A Mapping Study of Computational Thinking and Programming in Brazilian K-12 Education". In: Proc. of the 48th Annual Frontiers In Education Conference, San Jose, EUA.

Valente, J. A. (2016). Integração do Pensamento Computacional no Currículo da Educação Básica: diferentes estratégias usadas e questões de formação de professores e avaliação do aluno. PUC, São Paulo.

waiselfisz, Julio Jacobo. Lápis, borracha e teclado: tecnologia da informação na educação. Brasília: Rede de Informação Tecnológica Latino-americana (ritla), 2007.

Wing, J. M. (2006). “Computational thinking”, Communications of the ACM, v. 49, n. 3, p. 33 